\title{
Low serum bilirubin level predicts the develop- ment of chronic kidney disease in patients with type 2 diabetes mellitus
}

Kang Hee Ahn ${ }^{1,}$, Sang Soo Kim, ${ }^{1,2}$, Won Jin Kim ${ }^{1}$, Jong Ho Kim ${ }^{1}$, Yun Jeong Nam ${ }^{1}$, Su Bin Park ${ }^{1}$, Yun Kyung Jeon ${ }^{1}$, Bo Hyun $\mathrm{Kim}^{1}$, In Joo Kim${ }^{1,2}$, and Yong Ki Kim³

${ }^{1}$ Division of Endocrinology and Metabolism, Department of Internal Medicine, ${ }^{2}$ Biomedical Research Institute, Pusan National University Hospital, Busan; ${ }^{3}$ Kim Yong Ki Internal Medicine Clinic, Busan, Korea

Received: June 3, 2015

Revised : October 8, 2015

Accepted: May 17, 2016

\section{Correspondence to}

In Joo Kim, M.D.

Department of Internal Medi-

cine, Pusan National University

Hospital, 179 Gudeok-ro, Seo-gu,

Busan 49241, Korea

Tel: +82-51-240-7224

Fax: +82-51-254-3127

E-mail:injkim@pusan.ac.kr

*These authors contributed equally to this work.
Background/Aims: We evaluated whether serum bilirubin levels can predict the development of chronic kidney disease (CKD) in patients with type 2 diabetes mellitus (T2DM).

Methods: This was a retrospective observational longitudinal study of patients presenting at the Pusan National University Hospital. A total of 349 patients with $\mathrm{T} 2 \mathrm{DM}$ and preserved kidney function (estimated glomerular filtration rate $\geq 60$ $\mathrm{mL} / \mathrm{min} / 1.73 \mathrm{~m}^{2}$ ) were enrolled. The main outcome was the development of CKD stage 3 or greater. The patients were divided into four groups according to the quartiles of the total serum bilirubin levels at baseline.

Results: The group with the lowest range of total serum bilirubin level (Q1) showed the highest cumulative incidence of CKD stage 3 or greater than that of the other lower quartiles (Q1 vs. Q4; hazard ratio [HR], 6.75; 95\% confidence interval [CI], 1.54 to $29.47 ; p=0.011$ ). In multivariate analysis, the risk of developing CKD stage 3 or greater was higher in the second lowest quartile of the serum bilirubin level than that in the highest quartile of the serum bilirubin level (Q2 vs. Q4; HR, 9.36; $95 \%$ CI, 1.33 to $65.73 ; p=0.024$ ). In the normoalbuminuria subgroup $(n=236)$, multivariate analysis showed that the risk of developing CKD stage 3 or greater was higher in the lowest quartile of the serum bilirubin level than that in the highest quartile of the serum bilirubin level (Q1 vs. Q4; HR, 7.36; 95\% CI, 1.24 to $35.82 ; p=0.019$ ).

Conclusions: Serum bilirubin might be an early clinical marker for predicting the progression of CKD in patients with $\mathrm{T} 2 \mathrm{DM}$ and preserved renal function.

Keywords: Diabetic nephropathies; Bilirubin; Diabetes mellitus, type 2; Renal insufficiency, chronic

\section{INTRODUCTION}

Korean subjects with diabetes have a higher prevalence of albuminuria and chronic kidney disease (CKD) than those without diabetes [1]. Diabetic nephropathy (DN), which is a major end-organ complication in diabetes, continues to be the most common cause of end-stage renal disease (ESRD), and accounts for $>40 \%$ of patients on renal replacement therapy [2]. With the increasing number of patients with type 2 diabetes mellitus ( $\mathrm{T} 2 \mathrm{DM})$, the increase in the number of patients with CKD and ESRD has become a social burden [3]. Oxida- 
tive stress is a potential factor in the pathogenesis of diabetic vascular complications, including DN [4].

Bilirubin is a natural product of heme catabolism by heme oxygenase, a key antioxidant enzyme, and is excreted by liver cells [5]. It is a potent anti-oxidant that effectively scavenges peroxyl radicals, and suppresses the oxidation of lipids and lipoproteins [6]. In diabetic patients with Gilbert syndrome, a common hereditary disorder (incidence of $5 \%$ to $10 \%$ of the population) characterized by high levels of unconjugated bilirubin, vascular complications, including $\mathrm{DN}$, were infrequent [7]. In a cross-sectional study, serum bilirubin levels were negatively correlated with urinary albumin excretion, and positively correlated with the glomerular filtration rate (GFR) in patients with $\mathrm{T} 2 \mathrm{DM}[8,9]$. However, the role of serum bilirubin in the development and progression of CKD in patients with T2DM with preserved kidney function has not been investigated.

We determined whether total serum bilirubin levels can predict the development of CKD in patients with $\mathrm{T} 2 \mathrm{DM}$ with preserved kidney function at baseline.

\section{METHODS}

\section{Patients}

This was a retrospective longitudinal observational study of patients presenting to the Department of Endocrinology and Metabolism, Pusan National University Hospital. This study protocol was approved by the Institutional Research Board of Pusan National University Hospital (E-2015116). A total of 618 outpatients with T2DM were enrolled between January 2008 and December 2009. The patients were followed up until August 2014. Of the 618 patients, 478 fulfilled the following inclusion criteria.

Estimated glomerular filtration rate (eGFR) $\geq 60 \mathrm{~mL} /$ $\min / 1.73 \mathrm{~m}^{2}$. Patients were excluded if their serum bilirubin levels were $>1.2 \mathrm{mg} / \mathrm{dL}$ because of the potential for confounding hepatobiliary or hemolytic diseases. Patients were also excluded if they had gallstones, anemia, cirrhosis, hepatitis B or C, alcoholic liver disease, or malignant disease, if they had undergone renal replacement therapy, or if they were pregnant. Of patients taking a drug that can influence the level of bilirubin were also ruled out as follows: antibiotics like penicil- lin G, sedatives like phenobarbital, diuretics like furosemide, and asthma medications like theophylline. Of the 478 patients, 102 were excluded because of lack of a baseline urine albumin to creatinine ratio (ACR). Among the 376 patients remaining, seven were excluded because of a newly diagnosed neoplasm, and 20 were excluded because of acute and chronic disease requiring hospital admission ( $n=14)$ or death $(n=6)$. Finally, 349 patients with $\mathrm{T} 2 \mathrm{DM}$, preserved kidney function (eGFR $\geq 60 \mathrm{~mL} /$ $\min / 1.73 \mathrm{~m}^{2}$ ) were included. Their age was 20 to 86 years. Type 1 diabetes mellitus and secondary diabetes mellitus patients were excluded.

\section{Measurements}

eGFR was estimated by the Modification of Diet in Renal Disease $(M D R D)$ study formula: $M D R D=175 \times($ serum creatinine $[\mathrm{mg} / \mathrm{dL}])^{-1.154} \times$ age $^{-0.203}[10]$. A correction factor of 0.742 was used for females. Renal function was conducted at least once a year. CKD stage 3 or greater was defined as GFR $<60 \mathrm{~mL} / \mathrm{min} / 1.73 \mathrm{~m}^{2}$. Serum bilirubin levels were measured using an enzymatic method involving bilirubin oxidase and an automatic analyzer (Hitachi Labospect oo8, Hitachi, Japan; normal range, 0.2 to $1.2 \mathrm{mg} / \mathrm{dL}$ ). The ACR was measured from spot urine. Albuminuria values were defined as follows: normoalbuminuria was defined as ACR $<30 \mathrm{mg} / \mathrm{g}$, microalbuminuria was defined as ACR 30 to $299 \mathrm{mg} / \mathrm{g}$, and macroalbuminuria was defined as ACR $\geq 300 \mathrm{mg} / \mathrm{g}$ [11]. The main outcome was the development of CKD stage 3 or greater.

\section{Statistical analyses}

Statistical analyses were performed using SPSS version 18.0 (SPSS Inc., Chicago, IL, USA). Data are presented as mean \pm standard deviation for normally distributed variables and medians (interquartile range) for nonparametric variables. Categorical data are expressed as frequencies and percentages. Analysis of variance was used to evaluate differences among the total serum bilirubin quartile groups. The cumulative incidence of CKD stage 3 or greater was evaluated using the Kaplan-Meier method and log-rank test. Univariate analysis was performed to determine the relationship between the development of CKD stage 3 or greater and the variables. Multivariate analysis was conducted using a stepwise procedure. Cox regression analysis was performed to assess the effect of 
multiple variables on the development of CKD stage 3 or greater. Results are presented as hazard ratios (HRs) and 95\% confidence intervals (CIs). A p value less than 0.05 was considered statistically significant.

\section{RESULTS}

Table 1 summarizes the baseline characteristics of the patients according to the total serum bilirubin level quartiles. The mean serum bilirubin level was $0.75 \pm 0.29$ $\mathrm{mg} / \mathrm{dL}$. The mean age of the patients was $55 \pm 11.7$ years (range, 20 to 86). They consisted of 136 males (39\%) and 213 females $(61 \%)$. Sex, age, body mass index, the presence of hypertension, systolic blood pressure, diastolic blood pressure, the total cholesterol level, the low density lipoprotein cholesterol level, the high density lipoprotein cholesterol level, the triglyceride level, eGFR, the use of aspirin, and the use of lipid-lowering agents, medication of diabetes were not significantly different

Table 1. Baseline characteristics of patients with type 2 diabetes mellitus and preserved kidney function according to serum bilirubin quartile

\begin{tabular}{|c|c|c|c|c|c|}
\hline Characteristic & $\mathrm{Q}_{1}(\leq 0.55)$ & Q2 $(0.55-0.71)$ & Q3 $(0.71-0.88)$ & $\mathrm{Q}_{4}(>0.88)$ & $p$ value \\
\hline Number & 89 & 89 & 84 & 87 & \\
\hline Sex, male/female & $27 / 62$ & $32 / 57$ & $34 / 50$ & $43 / 44$ & 0.065 \\
\hline Age, yr & $55.4 \pm 11.5$ & $55.8 \pm 10.8$ & $54.2 \pm 11.3$ & $54.6 \pm 13.0$ & 0.801 \\
\hline BMI, kg/m² & $24.2 \pm 2.9$ & $24.9 \pm 3.4$ & $24.1 \pm 3 \cdot 3$ & $24.5 \pm 3.0$ & 0.350 \\
\hline Duration of diabetes, yr & $9.1 \pm 6.0$ & $8.7 \pm 5.4$ & $8.7 \pm 6.2$ & $11.7 \pm 7.3$ & 0.130 \\
\hline Hypertension & $43(48.3)$ & $26(29.2)$ & $38(45.2)$ & $41(47.1)$ & 0.092 \\
\hline $\mathrm{SBP}, \mathrm{mmHg}$ & $124 \pm 15$ & $125 \pm 13$ & $122 \pm 17$ & $124 \pm 15$ & 0.562 \\
\hline $\mathrm{DBP}, \mathrm{mmHg}$ & $74 \pm 11$ & $76 \pm 10$ & $75 \pm 10$ & $77 \pm 12$ & 0.225 \\
\hline HbAic, \% & $7.8 \pm 1.5$ & $7 \cdot 3 \pm 1.2$ & $7 \cdot 3 \pm 1.4$ & $7.6 \pm 1.6$ & 0.046 \\
\hline Total cholesterol, mg/dL & $177 \pm 40$ & $186 \pm 43$ & $181 \pm 45$ & $178 \pm 42$ & 0.845 \\
\hline $\mathrm{LDL}-\mathrm{C}, \mathrm{mg} / \mathrm{dL}$ & $95 \pm 33$ & $106 \pm 36$ & $101 \pm 35$ & $100 \pm 33$ & 0.208 \\
\hline HDL-C, mg/dL & $48 \pm 16$ & $45 \pm 11$ & $49 \pm 12$ & $48 \pm 12$ & 0.158 \\
\hline Triglycerides, mg/dL & $176(145-206)$ & $173(150-194)$ & $161(139-182)$ & $146(119-171)$ & 0.324 \\
\hline Serum creatinine, $\mathrm{mg} / \mathrm{dL}$ & $0.84 \pm 0.17$ & $0.82 \pm 0.13$ & $0.83 \pm 0.17$ & $0.82 \pm 0.19$ & 0.644 \\
\hline $\mathrm{eGFR}, \mathrm{mL} / \mathrm{min} / 1.73 \mathrm{~m}^{2}$ & $84.6 \pm 19 \cdot 3$ & $87.4 \pm 14.7$ & $89.6 \pm 16.8$ & $93.6 \pm 19.2$ & 0.008 \\
\hline Urine ACR, mg/g & $114.2(58-170)$ & $122.9(39-206)$ & $32.3(19-45)$ & $65 \cdot 3(20-110)$ & 0.085 \\
\hline Total bilirubin, mg/dL & $0.45 \pm 0.07$ & $0.64 \pm 0.05$ & $0.80 \pm 0.05$ & $1.15 \pm 0.27$ & $<0.001$ \\
\hline Hemoglobin, g/dL & $12.6 \pm 1.5$ & $13.4 \pm 1.3$ & $14.1 \pm 1.4$ & $14.1 \pm 1.6$ & $<0.001$ \\
\hline Diabetic retinopathy & $34(38.2)$ & $19(21.3)$ & $17(20.2)$ & $20(23.0)$ & 0.996 \\
\hline Aspirin & $27(30.3)$ & $19(21.3)$ & $27(32.1)$ & $22(25 \cdot 3)$ & 0.141 \\
\hline Lipid-lowering agent & $45(50.6)$ & $45(50.6)$ & $54(64 \cdot 3)$ & $40(46.0)$ & 0.080 \\
\hline RAS inhibitor & $46(51.7)$ & $49(55.1)$ & $39(46.4)$ & $45(51.7)$ & 0.256 \\
\hline Medication of diabetes & & & & & 0.985 \\
\hline None & $2(2.2)$ & $4(4 \cdot 5)$ & $3(3.6)$ & $3(3 \cdot 5)$ & \\
\hline OHA & $58(65.2)$ & $62(69.7)$ & $60(71.4)$ & $62(71.3)$ & \\
\hline Insulin & $6(6.7)$ & $5(5.6)$ & $4(4.8)$ & $5(5.8)$ & \\
\hline OHA + Insulin & $23(25.8)$ & $18(20.2)$ & $17(20.2)$ & $17(19 \cdot 5)$ & \\
\hline
\end{tabular}

Values are presented as mean $\pm \mathrm{SD}$, number (\%), or median (interquartile range).

BMI, body mass index; SBP, systolic blood pressure; DBP, diastolic blood pressure; HbA1c, glycated hemoglobin; LDL-C, low density lipoprotein cholesterol; HDL-C, high density lipoprotein cholesterol; eGFR, estimated glomerular filtration rate; ACR, albumin to creatinine ratio; RAS, renin-angiotensin system; OHA, oral hypoglycemic agent. 
between the groups. The group with the highest total serum bilirubin level had higher eGFR, and a higher hemoglobin level ( $p=0.008$ and $p<0.001$, respectively). The median follow-up duration was 41 months (range, 5.0 to 70.0) (Table 1).

Of the 349 patients, 33 (9.5\%) progressed to CKD 3 or greater during the follow-up period. Of the 33 patients, 28 (84.8\%) progressed to CKD 3, three (9.1\%) progressed to $\mathrm{CKD} 4$, and two (6.1\%) progressed to CKD 5. A significant difference was observed in the cumulative incidence of CKD stage 3 or greater (GFR $<60 \mathrm{~mL} / \mathrm{min} / 1.73$ $\mathrm{m}^{2}$ ) using the Kaplan-Meier method and log-rank test in patients according to the total serum bilirubin level quartile $(p<0.001)$. The group with the lowest range of total serum bilirubin (Q1) showed a higher cumulative incidence of CKD stage 3 or greater than that of the other quartiles (Fig. 1).

A longer duration of diabetes (HR, 1.13; $p=0.002)$, age (HR, 1.04; $p=0.020$ ), hemoglobin level (HR, 0.75; $p$ $=0.021$ ), lowest total bilirubin level (Q1) (HR, 6.75; $p=$ 0.011 ), and renin-angiotensin system (RAS) inhibitor use $(\mathrm{HR}, 2.38 ; p=0.027)$ were significantly associated with the development of $\mathrm{CKD}_{3}$ or greater in univariate analysis.

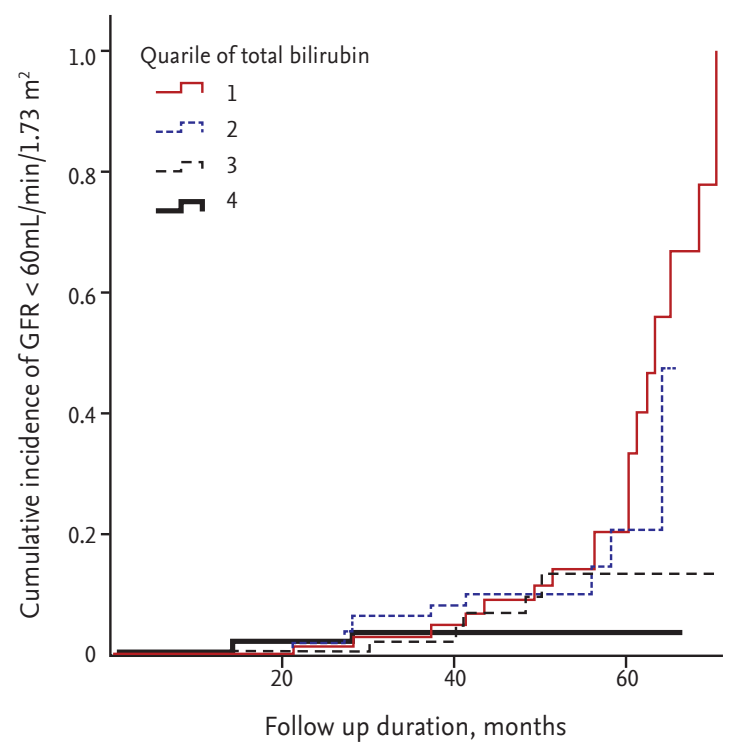

Figure 1. The cumulative incidence of chronic kidney disease (CKD) stage 3 or greater (estimated glomerular filtration rate $\left[\right.$ eGFR] < $60 \mathrm{~mL} / \mathrm{min} / 1.73 \mathrm{~m}^{2}$ ) using the Kaplan-Meier method and log-rank test in patients with type 2 diabetes mellitus according to total bilirubin level quartile. Quartile 1 (Q1) shows a higher cumulative incidence of CKD stage 3 or greater than the other quartiles.
In multivariate analysis, male sex (HR, 5.57; 95\% CI, 1.44 to $21.50 ; p=0.013)$, age (HR, 1.11; $95 \% \mathrm{CI}, 1.04$ to $1.19 ; p$ $=0.002$ ), duration of diabetes (HR, 1.19; $95 \% \mathrm{CI}, 1.08$ to 1.31; $p=0.001$ ), hemoglobin level (HR, 0.58; 95\% CI, 0.34 to $0.97 ; p=0.039)$, and the second lowest total bilirubin level (Q2; HR, 9.36; 95\% CI, 1.33 to 65.72; $p=0.024$ ) were significantly associated with CKD 3 or greater (Table 2).

In the normoalbuminuria subgroup $(n=236)$, univariate analysis showed that age was significantly associated with the development of CKD 3 or greater in univariate analysis (HR, 1.05; $p=0.037)$. In multivariate analysis, age and the lowest total bilirubin level (Q1) was significantly associated with the development of CKD 3 or greater (HR, 1.09; 95\% CI, 1.002 to 1.194; $p=0.046)(\mathrm{HR}, 7.36$; $95 \%$ CI, 1.24 to $35.82 ; p=0.019$ ) (Table 3 ).

We analyzed progression from normoalbuminuria to microalbuminuria, microalbuminuria to macroalbuminuria according to bilirubin quartile in univariated and multivariated model, but there are no statistically significant linear trends across bilirubin quartile. Multivariable adjusted odds ratios for progression from normoalbuminuria to microalbuminuria for the second, third, and fourth quartile of serum bilirubin levels were 1.12 (95\% CI, 0.52 to 2.14), 0.95 (95\% CI, 0.57 to 1.72), and 0.89 (95\% CI, 0.42 to 1.68), respectively, showing no statistically significant linear trend across categories $(p$ $=0.152$ ). Multivariable adjusted odds ratios for progression from microalbuminuria to macroalbuminuria for the second, third, and fourth quartile of serum bilirubin levels were 0.93 (95\% CI, 0.47 to 1.82), 1.24 (95\% CI, 0.56 to 2.84), and 1.07 (95\% CI, 0.59 to 1.93), respectively, showing no statistically significant linear trend across categories $(p=0.210)$.

\section{DISCUSSION}

We found that a low serum bilirubin level was an independent predictor of development of CKD stage 3 or greater in patients with T2DM with preserved kidney function. A low serum bilirubin level is a risk factor for the development of CKD of stage 3 or greater in univariate and multivariate analyses. An association between bilirubin level and development of CKD stage 3 or greater was also observed in the subgroup of patients with normoalbuminuria. These significant correlations per- 
Table 2. Univariate and multivariate analysis for the development of chronic kidney disease stage 3 or greater (eGFR < $60 \mathrm{~mL} /$ $\min / 1.73 \mathrm{~m}^{2}$ ) in patients with type 2 diabetes mellitus and preserved kidney function

\begin{tabular}{|c|c|c|c|c|c|}
\hline \multirow{2}{*}{ Variable } & \multicolumn{2}{|c|}{ Univariate analysis } & \multicolumn{3}{|c|}{ Multivariate analysis } \\
\hline & HR & $p$ value & $\mathrm{HR}$ & $95 \% \mathrm{CI}$ & $p$ value \\
\hline Male sex & 1.42 & 0.325 & $5 \cdot 57$ & $1.44-21.50$ & 0.013 \\
\hline Age, yr & 1.04 & 0.020 & 1.11 & $1.04-1.19$ & 0.002 \\
\hline $\mathrm{BMI}, \geq 25 \mathrm{~kg} / \mathrm{m}^{2}$ & 0.06 & 0.465 & 0.14 & $0.82-1.13$ & 0.382 \\
\hline Duration of diabetes, yr & 1.13 & 0.002 & 1.19 & $1.08-1.31$ & 0.001 \\
\hline Hypertension, yes & 1.58 & 0.193 & 1.03 & $0.36-2.90$ & 0.962 \\
\hline $\mathrm{SBP}, \mathrm{mmHg}$ & 1.01 & 0.342 & 0.89 & $0.73-1.23$ & 0.678 \\
\hline $\mathrm{HbA1c}, \geq 7 \%$ & 1.32 & 0.447 & 0.58 & $0.34-0.97$ & 0.039 \\
\hline Hemoglobin & 0.75 & 0.021 & 0.58 & $0.37-0.87$ & 0.072 \\
\hline $\mathrm{LDL}-\mathrm{C}, \geq 100 \mathrm{mg} / \mathrm{dL}$ & 1.58 & 0.209 & 2.14 & $1.35-1.67$ & 0.725 \\
\hline HDL-C $, \leq 40 \mathrm{mg} / \mathrm{dL}(\mathrm{M}), \leq 50 \mathrm{mg} / \mathrm{dL}(\mathrm{F})$ & 1.04 & 0.908 & 1.62 & $1.12-1.53$ & 0.438 \\
\hline Triglycerides, $\geq 150 \mathrm{mg} / \mathrm{dL}$ & 1.34 & 0.423 & 1.21 & $1.32-1.84$ & 0.376 \\
\hline \multicolumn{6}{|l|}{ Total bilirubin } \\
\hline Q1 & 6.75 & 0.011 & 3.20 & $0.51-20.11$ & 0.215 \\
\hline Q2 & $4 \cdot 57$ & 0.053 & $9 \cdot 36$ & $1.33-65.73$ & 0.024 \\
\hline Q3 & 2.40 & 0.297 & 2.47 & $0.23-26.51$ & 0.454 \\
\hline $\mathrm{Q}_{4}$ & 1.00 & 0.027 & 1.00 & - & 0.039 \\
\hline Diabetic retinopathy, yes & 1.85 & 0.099 & 1.12 & $0.62-1.42$ & 0.524 \\
\hline Lipid-lowering agent, yes & 0.69 & 0.296 & 0.83 & $0.52-1.24$ & 0.243 \\
\hline RAS inhibitor, yes & 2.38 & 0.027 & 1.45 & $0.44-4.78$ & 0.545 \\
\hline
\end{tabular}

Quartiles of serum total bilirubin are as follows: $\leq 0.55,0.55-0.71,0.71-0.88$, and $>0.88$.

eGFR, estimated glomerular filtration rate; HR, hazard ratio; CI, confidence interval; BMI, body mass index; SBP, systolic blood pressure; HbA1c, glycated hemoglobin; LDL-C, low density lipoprotein cholesterol; HDL-C, high density lipoprotein cholesterol; RAS, renin-angiotensin system.

sisted after additional analysis with hemoglobin level in the multivariable statistical model. Contrast to expectation, the patients who took RAS inhibitors at baseline had higher risk for development of CKD 3 or greater in univariate analysis. However, the use of RAS inhibitors was not associated with CKD progression in multivariate analysis. As the patients who already had had albuminuria at baseline were prescribed RAS inhibitors, it is considered that those patients easily progressed into CKD 3 or greater. This assumption is supported by the results that the use of RAS inhibitors was not associated with the CKD progression in the analysis with normoalbuminuric patients. To the best of our knowledge, this is the first study to investigate the association between the development of CKD 3 or greater and serum bilirubin level in patients with T2DM with preserved kidney function.
Chronic hyperglycemia results in the generation of reactive oxygen species (e.g., superoxide), predominantly by the mitochondria. This pathway is particularly prominent in endothelial cells and leads to vascular dysfunction, a major underlying feature in both diabetes and its complications [4]. Increased oxidative stress was associated with the development of diabetic microvascular complications [12-14], and oxidative stress in the kidney is increased in diabetic conditions compared to the control [15]. Sasaki and Inoguchi [16] reported that the markers of oxidative stress such as urinary 8-hydroxy-2'-deoxyguanosine were reduced in diabetic patients with Gilbert syndrome. Fujii et al. [13] showed that bilirubin and biliverdin, the precursor of bilirubin, protect against DN through the inhibition of nicotinamide adenine dinucleotide phosphate-dependent superoxide production. Riphagen et al. [17] found a negative correla- 
Table 3. Univariate and multivariate analysis for the development of chronic kidney disease stage 3 or greater (eGFR < $60 \mathrm{~mL} /$ $\min / 1.73 \mathrm{~m}^{2}$ ) in the normoalbuminuria subgroup

\begin{tabular}{|c|c|c|c|c|c|}
\hline \multirow{2}{*}{ Variable } & \multicolumn{2}{|c|}{ Univariate analysis } & \multicolumn{3}{|c|}{ Multivariate analysis } \\
\hline & HR & $p$ value & HR & $95 \% \mathrm{CI}$ & $p$ value \\
\hline Male sex & 1.48 & 0.341 & 2.49 & $0.76-8.83$ & 0.159 \\
\hline Age, yr & 1.05 & 0.037 & 1.09 & $1.002-1.19$ & 0.046 \\
\hline $\mathrm{BMI}, \geq 25 \mathrm{~kg} / \mathrm{m}^{2}$ & 0.38 & 0.056 & 0.65 & $0.58-1.03$ & 0.094 \\
\hline Duration of diabetes, yr & 0.95 & 0.594 & 0.83 & $0.66-1.05$ & 0.113 \\
\hline Hypertension, yes & 1.42 & 0.164 & 2.24 & $0.72-1.23$ & 0.243 \\
\hline $\mathrm{SBP}, \mathrm{mmHg}$ & 1.21 & 0.142 & 1.34 & $0.82-1.41$ & 0.564 \\
\hline $\mathrm{HbA} 1 \mathrm{c}, \geq 7 \%$ & 1.12 & 0.143 & 1.62 & $0.73-1.24$ & 0.728 \\
\hline Hemoglobin & 0.86 & 0.034 & 0.78 & $0.43-0.92$ & 0.125 \\
\hline $\mathrm{LDL}-\mathrm{C}, \geq 100 \mathrm{mg} / \mathrm{dL}$ & 1.92 & 0.309 & 1.67 & $0.69-1.84$ & 0.384 \\
\hline $\mathrm{HDL}-\mathrm{C}, \leq 40 \mathrm{mg} / \mathrm{dL}(\mathrm{M}), \leq 50 \mathrm{mg} / \mathrm{dL}(\mathrm{F})$ & 1.62 & 0.732 & 1.26 & $0.52-2.85$ & 0.826 \\
\hline Triglycerides, $\geq 150 \mathrm{mg} / \mathrm{dL}$ & 1.62 & 0.522 & 1.33 & $0.91-2.42$ & 0.423 \\
\hline \multicolumn{6}{|l|}{ Total bilirubin } \\
\hline Q1 & 3.77 & 0.094 & $7 \cdot 36$ & $1.24-35.82$ & 0.019 \\
\hline Q2 & 2.71 & 0.222 & $5 \cdot 37$ & $0.94-24.32$ & 0.060 \\
\hline Q3 & 0.85 & 0.872 & 0.95 & $0.35^{-1.04}$ & 0.959 \\
\hline Q4 & 1.00 & 0.148 & 1.00 & - & 0.040 \\
\hline Diabetic retinopathy, yes & 1.74 & 0.082 & 1.85 & $1.12-1.98$ & 0.099 \\
\hline Lipid-lowering agent, yes & 1.42 & 0.436 & 1.27 & $0.52-1.87$ & 0.362 \\
\hline RAS inhibitor, yes & 3.24 & 0.213 & 2.38 & $0.89-3.21$ & 0.545 \\
\hline
\end{tabular}

Quartiles of serum total bilirubin are as follows: $\leq 0.55,0.55-0.71,0.71-0.88$, and $>0.88$.

eGFR, estimated glomerular filtration rate; HR, hazard ratio; CI, confidence interval; BMI, body mass index; SBP, systolic blood pressure; HbA1c, glycated hemoglobin; LDL-C, low density lipoprotein cholesterol; HDL-C, high density lipoprotein cholesterol; RAS, renin-angiotensin system.

tion between baseline serum bilirubin levels and the doubling of serum creatinine levels and ESRD during a median follow-up of $\sim 3$ years for a prospective assessment of a post hoc analysis of the results of the Reduction of End Points in Non-Insulin-Dependent Diabetes Mellitus with the Angiotensin II Antagonist Losartan (RENAAL) trial [18] and the Irbesartan Diabetic Nephropathy Trial (IDNT) [19]. Toya et al. [20] showed that higher serum bilirubin levels, within the normal range, were associated with a lower risk of progression from microalbuminuria to macroalbuminuria in both univariate and multivariate analyses. However, the authors did not find a relationship between the baseline bilirubin level and rate of GFR decline. In the present study, we showed that the group with the lowest range of total serum bilirubin level (Q1) at baseline showed the highest cumulative incidence of the development CKD stage 3 or greater $\left(\mathrm{GFR}<60 \mathrm{~mL} / \mathrm{min} / 1.73 \mathrm{~m}^{2}\right)$ of the other lower quartiles. Mashitani et al. [21] showed a significant correlation between baseline serum bilirubin levels and DN progression after adjustment for multiple confounders. However, they could not show a significant correlation after adding hemoglobin levels to the multivariable model. In contrast to this study, we showed that the risk of developing CKD stage 3 or greater was higher in the second lowest quartile of the serum bilirubin levels than that in the highest quartile of the serum bilirubin levels in multivariate analysis, including hemoglobin levels. In addition, bilirubin has an anti-inflammatory property. Mazzone et al. [22] showed that bilirubin inhibits the tumor necrosis factor- $\alpha$-induced gene upregulation of endothelial adhesion molecules, including E-selectin, vascular cell adhesion molecule 1 (VCAM-1), and intercellular adhesion molecule 1 (ICAM-1). E-selectin may 
play a key role in leukocyte infiltration into the renal interstitium [23]. ICAM-1 and VCAM-1 are critically involved in the pathogenesis of DN [24]. The precise nature of the relationship between DN development and serum bilirubin level is unknown, but we expect that increased serum bilirubin levels can inhibit oxidative stress and inflammation; thereby, preventing DN development.

Our study had several limitations. First, this study was retrospective, and the records of patients were from a single institution. Second, the follow-up duration was 41 months (range, 5.0 to 70.0), which is relatively short. Third, the number of patients available for analysis was not large, and the number of patients who developed CKD stage 3 or greater was small in this study (9.5\%). We evaluated DN using single urine ACR measurements at baseline and during follow-up, which may have resulted in misclassification of DN. Large prospective trials are needed to better assess the relationship between the serum bilirubin concentration and CKD development in patients with T2DM. Despite these limitations, this study included many confounders known to be associated with the reduction in eGFR. Another strength of this study is that we enrolled patients with T2DM with preserved kidney function.

Bilirubin measurement is inexpensive, is performed routinely, and is accessible to most medical centers. Thus, serum bilirubin levels can be used easily by clinicians as one of the risk factors for the development of DN. Additional studies must be conducted to understand the full clinical potential of bilirubin as a marker in monitoring DN. Early detection of CKD is important. Patients with a low-normal total bilirubin concentration may be managed aggressively to delay the progression to kidney failure.

In conclusion, the serum bilirubin level might be an early clinical marker for predicting the progression of CKD in patients with T2DM with preserved renal function. Careful consideration is needed when managing patients with T2DM and a low-normal total bilirubin concentration. Further studies are required to determine whether the total bilirubin concentration is a potential therapeutic target for the prevention of CKD.

\section{KEY MESSAGE}

1. We examine whether total serum bilirubin levels can predict the development of chronic kidney disease (CKD) in patients with type 2 diabetes mellitus ( $\left.\mathrm{T}_{2} \mathrm{DM}\right)$ with preserved kidney function at baseline.

2. The group with the lowest range of total serum bilirubin (Q1) showed a higher cumulative incidence of CKD stage 3 or greater than that of the other quartiles.

3. In the normoalbuminuria subgroup, multivariate analysis shows that the lowest total bilirubin level (Q1) was significantly associated with the development of CKD 3 or greater.

4. Serum bilirubin level may be an early clinical marker for predicting the progression of CKD in patients with $\mathrm{T} 2 \mathrm{DM}$ with preserved renal function.

\section{Conflict of interest}

No potential conflict of interest relevant to this article was reported.

\section{Acknowledgments}

This work was supported by clinical research grant from Pusan National University Hospital in 2014.

\section{REFERENCES}

1. Ahn JH, Yu JH, Ko SH, et al. Prevalence and determinants of diabetic nephropathy in Korea: Korea National Health and Nutrition Examination Survey. Diabetes Metab J 2014;38:109-119.

2. American Diabetes Association. (9) Microvascular complications and foot care. Diabetes Care 2015;38 Suppl:S58S66.

3. Kim WJ, Kim SS, Bae MJ, et al. High-normal serum uric acid predicts the development of chronic kidney disease in patients with type 2 diabetes mellitus and preserved kidney function. J Diabetes Complications 2014;28:130134.

4. Giacco F, Brownlee M. Oxidative stress and diabetic complications. Circ Res 2010;107:1058-1070.

5. Arias IM. The excretion of conjugated bilirubin by the 
liver cell. Medicine (Baltimore) 1966;45:513-515.

6. Stocker R, Yamamoto Y, McDonagh AF, Glazer AN, Ames BN. Bilirubin is an antioxidant of possible physiological importance. Science 1987;235:1043-1046.

7. Inoguchi T, Sasaki S, Kobayashi K, Takayanagi R, Yamada T. Relationship between Gilbert syndrome and prevalence of vascular complications in patients with diabetes. JAMA 2007;298:1398-1400.

8. Fukui M, Tanaka M, Shiraishi E, et al. Relationship between serum bilirubin and albuminuria in patients with type 2 diabetes. Kidney Int 2008;74:1197-1201.

9. Zelle DM, Deetman N, Alkhalaf A, Navis G, Bakker SJ. Support for a protective effect of bilirubin on diabetic nephropathy in humans. Kidney Int 2011;79:686.

10. Stevens LA, Coresh J, Greene T, Levey AS. Assessing kidney function: measured and estimated glomerular filtration rate. N Engl J Med 2006;354:2473-2483.

11. Molitch ME, DeFronzo RA, Franz MJ, et al. Diabetic nephropathy. Diabetes Care 2003;26 Suppl 1:S94-S98.

12. Madsen-Bouterse SA, Kowluru RA. Oxidative stress and diabetic retinopathy: pathophysiological mechanisms and treatment perspectives. Rev Endocr Metab Disord 2008;9:315-327.

13. Fujii M, Inoguchi T, Sasaki S, et al. Bilirubin and biliverdin protect rodents against diabetic nephropathy by downregulating $\mathrm{NAD}(\mathrm{P}) \mathrm{H}$ oxidase. Kidney Int 2010;78:905-919.

14. Baynes JW. Role of oxidative stress in development of complications in diabetes. Diabetes 1991;40:405-412.

15. Sonta T, Inoguchi T, Matsumoto $S$, et al. In vivo imaging of oxidative stress in the kidney of diabetic mice and its normalization by angiotensin II type 1 receptor blocker. Biochem Biophys Res Commun 2005;330:415-422.

16. Sasaki S, Inoguchi T. The role of oxidative stress in the pathogenesis of diabetic vascular complications. Diabetes Metab J 2012;36:255-261.
17. Riphagen IJ, Deetman PE, Bakker SJ, et al. Bilirubin and protection against progression of diabetic nephropathy. Proceedings of the Kidney Week 2012: American Society of Nephrology 45th Annual Meeting; 2012 Oct 30-Nov 4; San Diego, CA.

18. Brenner BM, Cooper ME, de Zeeuw D, et al. Effects of losartan on renal and cardiovascular outcomes in patients with type 2 diabetes and nephropathy. N Engl J Med 2001;345:861-869.

19. Lewis EJ, Hunsicker LG, Clarke WR, et al. Renoprotective effect of the angiotensin-receptor antagonist irbesartan in patients with nephropathy due to type 2 diabetes. $\mathrm{N}$ Engl J Med 2001;345:851-860.

20. Toya K, Babazono T, Hanai K, Uchigata Y. Association of serum bilirubin levels with development and progression of albuminuria, and decline in estimated glomerular filtration rate in patients with type 2 diabetes mellitus. J Diabetes Investig 2014;5:228-235.

21. Mashitani T, Hayashino Y, Okamura S, Tsujii S, Ishii H. Correlations between serum bilirubin levels and diabetic nephropathy progression among Japanese type 2 diabetic patients: a prospective cohort study (Diabetes Distress and Care Registry at Tenri [DDCRT 5]). Diabetes Care 2014;37:252-258.

22. Mazzone GL, Rigato I, Ostrow JD, et al. Bilirubin inhibits the TNFalpha-related induction of three endothelial adhesion molecules. Biochem Biophys Res Commun 2009;386:338-344.

23. Hirata K, Shikata K, Matsuda M, et al. Increased expression of selectins in kidneys of patients with diabetic nephropathy. Diabetologia 1998;41:185-192.

24. Okada S, Shikata K, Matsuda M, et al. Intercellular adhesion molecule-1-deficient mice are resistant against renal injury after induction of diabetes. Diabetes 2003;52:25862593. 